\title{
Spatiotemporal Changes of Tissue Glycans Depending on Localization in Cardiac Aging
}

\section{Yoko Itakura}

Research Team for Geriatric Medicine (Vascular Medicine), Tokyo Metropolitan Institute of Gerontology, 35-2 Sakae-cho, Itabashi-ku, Tokyo 173-0015

\section{Yasuko Hasegawa}

Research Team for Geriatric Pathology (Aging and Carcinogenesis), Tokyo Metropolitan Institute of Gerontology, 35-2 Sakae-cho, Itabashi-ku, Tokyo 173-0015

\section{Yurika Kikkawa}

Laboratory of Stem Cell Biology, Department of Biosciences, Kitasato University School of Science, 1-151 Kitasato, Minami-ku, Sagamihara, Kanagawa, 252-0373

\section{Yuina Murakami}

Laboratory of Environmental Molecular Physiology, School of Life Sciences, Tokyo University of Pharmacy and Life Sciences, 1432-1 Horinouchi, Hachioji, Tokyo, 192-0392

\section{Chiaki Nagai-Okatani}

Cellular and Molecular Biotechnology Research Institute, National Institutes of Advanced Industrial

Science and Technology, 5 Central, Tsukuba, 1-1-1 Higashi, Tsukuba-city, Ibaraki 305-8565

\section{Norihiko Sasaki}

Research Team for Geriatric Medicine (Vascular Medicine), Tokyo Metropolitan Institute of Gerontology, 35-2 Sakae-cho, Itabashi-ku, Tokyo 173-0015

\section{Mariko Umemura}

Laboratory of Environmental Molecular Physiology, School of Life Sciences, Tokyo University of Pharmacy and Life Sciences, 1432-1 Horinouchi, Hachioji, Tokyo, 192-0392

\section{Yuji Takahashi}

Laboratory of Environmental Molecular Physiology, School of Life Sciences, Tokyo University of Pharmacy and Life Sciences, 1432-1 Horinouchi, Hachioji, Tokyo, 192-0392

\section{Tohru Kimura}

Laboratory of Stem Cell Biology, Department of Biosciences, Kitasato University School of Science, 1-151 Kitasato, Minami-ku, Sagamihara, Kanagawa, 252-0373

\section{Atsushi Kuno}

Cellular and Molecular Biotechnology Research Institute, National Institutes of Advanced Industrial Science and Technology, 5 Central, Tsukuba, 1-1-1 Higashi, Tsukuba-city, Ibaraki 305-8565

\section{Toshiyuki Ishiwata}

Research Team for Geriatric Pathology (Aging and Carcinogenesis), Tokyo Metropolitan Institute of Gerontology, 35-2 Sakae-cho, Itabashi-ku, Tokyo 173-0015 
Masashi Toyoda ( $\square$ mtoyoda@tmig.or.jp)

Research Team for Geriatric Medicine (Vascular Medicine), Tokyo Metropolitan Institute of Gerontology, 35-2 Sakae-cho, Itabashi-ku, Tokyo 173-0015

\section{Research Article}

Keywords: cardiac tissue, heart failure, aging, glycan profile, microvessel

Posted Date: October 18th, 2021

DOl: https://doi.org/10.21203/rs.3.rs-960240/v1

License: (c) (1) This work is licensed under a Creative Commons Attribution 4.0 International License. Read Full License 


\title{
Title
}

Spatiotemporal changes of tissue glycans depending on localization in cardiac aging

\author{
Authors \\ Yoko Itakura $^{1}$, Yasuko Hasegawa ${ }^{2}$, Yurika Kikkawa ${ }^{1,3}$, Yuina Murakami ${ }^{1,4}$, Chiaki Nagai-Okatani ${ }^{5}$, Norihiko Sasaki ${ }^{1}$, \\ Mariko Umemura ${ }^{4}$, Yuji Takahashi ${ }^{4}$, Tohru Kimura ${ }^{3}$, Atsushi Kuno ${ }^{5}$, Toshiyuki Ishiwata ${ }^{2}$, Masashi Toyoda $^{1 *}$
}

\begin{abstract}
Affiliations
${ }^{1}$ Research Team for Geriatric Medicine (Vascular Medicine), Tokyo Metropolitan Institute of Gerontology, 35-2

Sakae-cho, Itabashi-ku, Tokyo 173-0015, Japan.

${ }^{2}$ Research Team for Geriatric Pathology (Aging and Carcinogenesis), Tokyo Metropolitan Institute of Gerontology, 35-2 Sakae-cho, Itabashi-ku, Tokyo 173-0015, Japan.

${ }^{3}$ Laboratory of Stem Cell Biology, Department of Biosciences, Kitasato University School of Science, 1-15-1 Kitasato, Minami-ku, Sagamihara, Kanagawa, 252-0373, Japan.

${ }^{4}$ Laboratory of Environmental Molecular Physiology, School of Life Sciences, Tokyo University of Pharmacy and Life Sciences, 1432-1 Horinouchi, Hachioji, Tokyo, 192-0392, Japan.

${ }^{5}$ Cellular and Molecular Biotechnology Research Institute, National Institutes of Advanced Industrial Science and Technology, 5 Central, Tsukuba, 1-1-1 Higashi, Tsukuba-city, Ibaraki 305-8565, Japan.
\end{abstract}

*Corresponding Author: Masashi Toyoda, $\mathrm{PhD}$

Research Team for Geriatric Medicine (Vascular Medicine), Tokyo Metropolitan Institute of Gerontology, Sakae-cho 35-2, Itabashi-ku, Tokyo 173-0015, Japan.

Tel: +81-3-3964-3241; Fax: +81-3-3579-4776

E-mail: mtoyoda@tmig.or.jp 


\begin{abstract}
Heart failure is caused by various factors, making its underlying pathogenic mechanisms difficult to identify, and tends to worsen over time. Early diagnosis of cardiovascular disease is the key for treatment to promote healthy life. To detect the structural and functional molecular changes associated with cardiovascular disease, we focused on glycans, which reflect the type and state of cells. We investigated glycan localization in the cardiac tissue of normal mice and their alterations during aging using an evanescent-filed lectin microarray, a technique based on lectin-glycan interaction, and lectin staining. The glycan profiles in the left ventricle showed differences between the luminal side (medial) and the wall side (lateral) region. The former area was characterized by the presence of sialic acid residues. Moreover, age-related changes in glycan profiles were observed earlier in the medial region. The difference in the age-related decrease of $\alpha$-galactose stained with griffonia simplicifolia lectin-IB4 in different region of the left ventricle suggested spatiotemporal changes in microvessels. The glycan profile, which retains diverse glycan structures, is supported by many cell populations and maintains cardiac function. Glycan localization and changes are expected to be developed as a marker of the signs and symptoms of heart failure in the future.
\end{abstract}

Keywords: cardiac tissue, heart failure, aging, glycan profile, microvessel 


\section{Introduction}

Age is a major risk factor for cardiovascular diseases, including heart failure ${ }^{1}$. The prevalence of heart failure has been increasing with an aging society. In particular, it is difficult for the elderly to detect the signs of cardiac dysfunction with age and they often overlook physiological symptoms; thus, heart failure tends to get gradually worse over time. A characteristic of heart failure at old age is a decline in diastolic function, but normal contractile function. Additionally, there are some age-related diseases that increase the risk of developing cardiovascular diseases, such as aortic valve stenosis and atrial fibrillation ${ }^{2-5}$. Further, acute myocardial infarction and angina pectoris caused by narrowing or thrombus in major vessels, such as the coronary artery, induce various complications, including left ventricular free wall rupture, ventricular septal perforation, and papillary muscle rupture ${ }^{6-8}$. Elderly patients reportedly have aggravated symptoms accompanied with cardiovascular complications in COVID-19 ${ }^{9-11}$. The reasons are that the virus causes a vascular inflammation through a receptor called angiotensin-converting enzyme 2 , which is a glycoprotein on the cell surface, and destroys the vascular integrity of the elderly who have advanced arteriosclerosis 12-16. There are few efficient therapeutic agents for cardiac dysfunction in the elderly. The specific function of cardiac tissue is supported by a large number of changing cell populations with aging ${ }^{17}$. Recently, it has been reported that various diseases are induced by senescent cells ${ }^{18-20}$ and that senescent cells are related to cardiovascular aging and age-related cardiovascular diseases ${ }^{21}$. Clarifying tissue alterations during aging could be useful for early diagnosis and the development of effective therapies against age-related heart failure. However, suitable biomarkers for distinguishing healthy aging from the impaired organ function associated with heart failure are lacking. Therefore, it is important to elucidate the mechanisms underlying cardiovascular diseases, in terms of which cell populations change structure and function with aging, to develop optimal therapies.

Glycans are suitable candidates for spatiotemporal analysis of molecular changes. Cell surfaces are covered with various glycans, conjugated proteins, and lipids, and these glycans have shown marked differences with respect to cellular characteristics or functional changes. For instance, it was shown that $O$-mannosyl glycan on $\alpha$-dystroglycan

disappears on muscle cells, when the cells bind to the extracellular matrix in congenital muscular dystrophy ${ }^{22}$. Lectin microarray is one of the analytical approaches to monitor glycans ${ }^{23-25}$. Previously, we showed that the amount of sialic acid residues on cell surface proteins reduces during cellular senescence and human aging using lectin microarrays ${ }^{26}$. Moreover, Miura et al. reported that the amount of high-branched $\mathrm{N}$-glycans on plasma proteins in supercentenarians 
is higher than that in younger individuals ${ }^{27}$. Thus, alterations in glycan profiles could indicate both cellular senescence and human aging. A study on glycoprotein-extracted tissue slices using lectin microarrays reported a high detection level in a small tissue section by observing pathomorphological features ${ }^{28}$. The lectin microarray analysis following the collection of patient tissue extracts with antibody showed differences of glycan structures in cancerous and noncancerous areas ${ }^{29}$. Specific glycans on each mouse organ were also shown using laser microdissection and lectin microarray ${ }^{30}$. Additionally, a glycome atlas has been developed by Konishi et al. ${ }^{31}$ The combination of glycan data from animal tissues and information tools, such as lectin microarray, will be useful in understanding glycan level and localization changes in different organs ${ }^{32}$. However, the spatiotemporal changes remain unclear. Therefore, we focused on the alterations of glycoproteins in the heart as an indicator to differentiate between aging and cardiac diseases.

In this study, glycan levels and localization in serial sections of the hearts of female mice were investigated using a lectin microarray, and the glycan level was examined to monitor molecular changes during aging. We focused on aging in female mice because the prevalence of cardiac diseases in postmenopausal females is significantly high. In the future, age-related changes in certain molecules, such as glycans, and the corresponding tissue morphologies could be therapeutic and diagnostic targets for cardiac diseases associated with aging in humans.

\section{Results}

Glycan mapping of mouse cardiac tissues and alterations with aging. To investigate the characteristics of glycans in mouse cardiac tissues, eight parts of the short-axis slices from normal female mouse hearts, the left ventricular wall (No. 1 and 2; ventral sides, 3 and 4; dorsal sides), the papillary muscle (No. 5 and 6), and the ventricular septum (No. 7 and 8) were analyzed for 2-, 12-, and 24-month-old mice using lectin microarray (Fig. 1b). As a result, a broad variety of lectin signals were detected at each location for all ages (see online resource Supplementary Fig. S1, Supplementary Data. S1). The major signal intensities were observed for lectins, Calsepa, ACG, STL, LEL, and ConA.

Further, the glycan profiles of eight areas of the heart of 2-month-old mice were analyzed in detail using PCA. As a result, papillary muscle (No.6) and ventricular septum (No.8) were placed in regions II and III, which are defined by sialic acid-binding lectins (SNA, SSA, TJA-I, ACG, and MAL-I) and chitin-binding lectins (LEL, STL, 
UDA, and WGA) that also bind to sialic acid residues in a multivalent manner ${ }^{33}$ (Fig. 2a). These indicate that both areas were characterized by sialic acid residues. Another ventricular septum (No. 7) was positioned at around the center of PC1. Furthermore, the ventral left ventricular wall (No. 1 and 2) was mainly located in regions I and IV, which are defined by $O$-glycan-binding lectins, such as WFA, SBA, VVA, PNA, BPL, and GSL-IB4. These regions were also characterized by large-branched $\mathrm{N}$-glycan-binding lectins, such as PHA-L and ECA, indicating the presence of glycans recognized by these lectins. On the other hand, the dorsal left ventricular wall (No. 3 and 4) showed a similar trend to the ventral left ventricular wall, although there were large interindividual differences. These results suggest that glycan clusters extracted from cardiac tissues could be roughly distinguished between the "lateral" (No. 1-4) and "medial" (No. 5-8) regions of the left ventricle. In a cross section of the heart, the orientation of the myocardial layers can be seen. Here, based on the shape of the myocardial layer, the wall side of the left ventricle was designated as the "lateral" and the medial region of the heart as the "medial".

Next, we examined changes in glycan profiles among 2-, 12-, and 24-month-old mice. The glycan profiles of each group of left ventricular wall (No. 1-4) and medial area of left ventricle (No. 5-8) in the three age groups were clearly divided into regions II/III and I/IV, which were characterized by several specific lectins, respectively (see online resource Supplemental Fig. S2). Similar to the analysis of 2-month-old mice, all medial areas (No.5-8) among the three age groups were characterized by sialic acid-related lectins, such as SNA, SSA, TJA-I, ACG, STL, UDA, and WGA.

Moreover, data for a group of left ventricular wall (No. 1-4) and medial side (No. 5-8) at each age were analyzed by hierarchical clustering. As a result, glycan profiles between the left ventricular wall and medial area were clearly differentiated, similar to the results in the PCA analysis (Fig. 2b). These hierarchical clustering data also indicated the degree of variation in glycan profiles as "distance". In the left ventricular wall, the profiles at 2 and 12 months were close, while in the medial side, the profiles at 12 and 24 months were close. This indicates that the rate of change in glycan profiles occurred at an earlier stage in the medial side than in the left ventricular wall. It also shows that the change in glycan profiles on the medial side was greater than that in the left ventricular wall.

We examined in more detail the age-related changes in glycan profiles in various areas of the left ventricle. In all areas, the profiles changed from regions III/IV to I/II with aging (Fig. 2c). In particular, the glycan profiles of the medial side, such as No. 5, 6, and 8, severely changed with aging. In addition, GSL-IB4 was shown to be a 
characteristic lectin in young mice. These results suggested that glycan profiles of cardiac tissues changed at the same trend with aging, although the rate of change varied from area to area.

The change in glycan signature is accompanied with cardiac morphology changes related to aging. To confirm the alteration of cardiac morphologies in the medial area with aging, the thin section of the papillary muscle was stained with HE and WGA, which is a lectin known to stain the cellular membrane ${ }^{34}$. The number of nuclei slightly decreased and cells were enlarged in HE-stained sections in 24-month-old mice (Fig. 3a and see online resource Supplemental Fig. S3a; left ventricular wall and ventricular spectrum). In the sections stained with WGA, cellular size at 24 months was slightly enlarged and non-uniform in morphology, and the cellular edge was obscure compared with those of 2-month-old mice (Fig. 3b and see online resource Supplemental Fig. S3b).

On the medial side, including the papillary muscle, changes in sialic acid were notably observed in the lectin microarray data (Fig. 2). To clarify this phenomenon, we investigated the changes in lectin signals by sialidase treatment in the papillary muscle. In the samples obtained from 2-month-old mice and that were allowed to react with sialidase A, which digests the $\alpha 2-3$ and 2-6 linkages of sialic acid, the signal intensities of sialic acid-recognizing lectins (MAL-I, SNA, SSA, TJA-I, ACG, MAH, and WGA) were drastically decreased. On the other hand, the signal intensities of lectins (ECA, RCA120, and WFA) that recognize glycan residues appearing when sialic acids were removed were increased (Fig. 3c upper, Table 1). In contrast, in the 24-month-old mice, the signal intensities of ACG, WGA, ECA, RCA120, and WFA showed little change (Fig. 3c lower, Table 1). These results suggested that the glycoproteins in papillary muscles had abundant sialic acid residues and that the diversity of these residues was lost with aging.

To further validate the binding of sialic acid, the samples extracted from thin sections were incubated with $\alpha 2-3$ sialidase. In 2-month-old mice, the signal intensities of MAL-I, ACG, MAH, and WGA were decreased by $\alpha 2-$ 3sialidase, while the signal intensities of ECA, RCA120, WFA, and LTL (no binding to $\alpha 2-3$-linked sialylated structure, such as sialyl-Le ${ }^{\mathrm{x}}$ ) were increased (Fig. 3d upper, Table 1). In the 24-month-old mice, the change in the signal intensities of ACG, LTL, ECA, and RCA120 due to enzyme treatment was small (Fig. 3d lower, Table 1). On the other hand, the change in the signal intensities of WGA and WFA was almost the same as that at 2 months of age. 
These results indicated that the residues of both $\alpha 2-6$ and 2-3-linked sialic acid, and a sialyl-Le ${ }^{\mathrm{x}}$ glycan structure were contained in the papillary muscle and that the amounts of their sialic acid residues in 24-month-old mice were lower than those in 2-month-old mice. Furthermore, these suggested that the diversity of sialic acid clearly changed with aging.

Changes in glycan levels in microvessel with aging. Lectin microarray data showed that the signal intensity of GSLIB4 was specific in 2-month-old mice and that the rate of change in glycan profiles was different between the medial and lateral sides of the left ventricular wall (Fig. 2b and 2c). To examine changes in glycans bound to GSL-IB4, which is used as an endothelial cell marker, the sections were stained with GSL-IB4 and the anti- $\alpha$-SMA antibody, which is used as a marker for smooth muscles, indicating arteries and veins (see online resource Supplemental Fig. 4). The area stained with GSL-IB4 in the cardiac tissue, except for major vessels stained with the anti- $\alpha$-SMA antibody, was denoted as microvessel, and the microvessel density around the cardiomyocyte was evaluated. Tissue sections of 2-, 6-, 12-, and 24-month-old mice were compared for each area. As a result, the stained area with only GSL-IB4 of the papillary muscle was shown to decrease between 2 and 6 months of age. The stained area of papillary muscle at 24 months of age (6.0\%) was lower than that at 2 months of age (8.4\%; no significant difference) (Fig. 4a). On the contrary, the stained area in the left ventricular wall (lateral areas) decreased markedly between the ages of 6 and 12 months $(2,6,12$, and 24 months; 7.4\%, 8.0\%, 5.6\%, and 5.6\%, respectively; no significant difference) (Fig. 4b). These results showed that the decrease of $\alpha$-galactose residues indicated by GSL-IB4 at the inner left ventricle, such as the papillary muscle, occurred at an earlier stage than that at the lateral left ventricle in cardiac tissue. This data suggested that microvessel changes on two dimensions are consistent with lectin microarray analysis.

\section{Discussion}

The risk of developing cardiovascular disease clearly increases in the elderly with frailty ${ }^{35,36}$. Cellular senescence around in cardiovascular system causes arteriosclerosis, leading to the accumulation of various molecules ${ }^{37,38}$. Thus, senescent cells are involved in various cardiovascular diseases, so identifying the molecular changes in the normal heart during aging is meaningful for diagnosing and treating cardiac dysfunction. Cardiac extracellular matrix (ECM) are also involved in various cardiac diseases from both structural and non-structural aspects ${ }^{39}$. Glycans on ECM and 
glycolipids are important not only for structural maintenance but also for functional aspects such as signal transduction. The major carriers of glycans on ECM are glycoproteins and proteoglycans, but lectin microarrays focus on glycoproteins due to the specificity of lectins and protein labeling methods. It is quite possible that some of those glycoproteins are not only cellular but also extracellular glycoproteins such as collagen and secretory proteins. Therefore, the results of tissue staining with lectin may not necessarily match the results of lectin microarray analysis. However, in fact, cell-specific glycans in tissue were stained by lectin and reflected in the signal by lectin microarray 40.

Understanding the spatiotemporal changes in glycans and their role in tissue alteration is important in some organs during aging or disease. For instance, glycans, such as disialyl-T antigen, are present at high levels, decreasing the core fucose levels in the tissues and serum of rats with cardiac hypertrophy ${ }^{41}$. In the hippocampus, the level of gangliosides, including $\alpha 2-3$ and 2-6 sialylated glycolipids, also decreases in the synaptic membrane during aging ${ }^{42}$. These glycans can be used as 'medical indicators'. We particularly focused on the papillary muscle, which is connected to the mitral valve and has an important role in mitral regulation ${ }^{43,44}$. In this study, it was suggested that sialic acid residues on the papillary muscle decrease with cardiac hypertrophy during aging. A previous report indicated that the amount of sialic acid on membrane proteins of human skin fibroblasts decreases with both cellular senescence and human aging, and that the reduction in the number of sialic acid residues suppressed activation of skin fibroblasts 26,45,46. Moreover, mesenchymal stem cells differentiated with a reduction of $\alpha 2-6$-linked sialic acid residue in vitro ${ }^{47}$. In these glycan changes, sialic acid residues were often related to aging and function. On the contrary, it was reported that the levels of sialic acid residues of cardiomyocytes from 6-month-old female rats were higher than those of neonatal rats ${ }^{40}$. Considering that these reports focused on different age groups, our results suggest that the amount of sialic acid in cardiac tissue from normal mice changes during aging, and it is predicted that sialic acid in cardiac cells also has a functional role in the heart. However, fibrosis in mouse cardiac tissue, detected via WGA staining, increases with aging or myocardial infarction ${ }^{48,49}$. WGA binds to sialic acid residues and chitin oligomers ${ }^{33}$. Further investigation is required to understand this, and caution should be taken when interpreting such data.

The present study also revealed that glycan profiles analyzed at each region were similar across all age groups. However, the reduction of $\alpha$-galactose residues indicated by GSLI-B4 differed among different cardiac regions. The reduction of $\alpha$-galactose residues, suggesting microvessel, in the papillary muscle occurred at an earlier stage than 
that in the lateral side of the left ventricular wall (Fig. 4). These alterations matched well with the change in glycan profiles in the lectin microarray data (Fig. $2 b$ and c). The changes in microvessel were not significant because the changes may have been due to normal aging. In pathological conditions, such as pressure on blood vessels due to high blood pressure or diabetes, microvessel changes show a significant difference. Microvessel reduction would inhibit the supply of oxygen to cells and initiate cellular necrosis or undernutrition, causing the reduction of vascularity in various diseases. The difference in reduced speed of the existence of microvessels between the medial and lateral sides of the left ventricle is considered to facilitate most heart failure cases in the elderly, which involves diastolic heart failure at different ages. It should be noted that the detection of the $\alpha$-galactose residue, which is the ligand of GSLIB4, is limited in specific non-primates, such as mice. The reduction of the glyco-epitope in mice has the potential to help elucidate the alteration in human microvessel. Moreover, mouse vascular endothelial cells can transform into smooth muscle cells or myofibroblasts via endothelial-to-mesenchymal transition (EMT) ${ }^{50}$. This reduction of $\alpha-$ galactose residues indicates that endothelial cells may undergo EMT and produce extracellular matrix, leading to increased fibrosis with aging. We predict that a series of these alterations may affect the maintenance of cardiac constituent cells. Previously, we found that the glycan profile of glycoproteins in human cardiomyocytes is similar to that in cardiac fibroblasts, except for some specific signatures (unpublished data). The glycan profiles of human cardiac cells showed only a small change in cellular senescence without a significant difference. Although the heart structure changed insignificantly at the cellular level, the changes seemed to occur due to interactions of multiple cells in the heart tissue. Although sialic acid changes observed during development are different, glycan changes on the cell surface regulate cell-ECM and are accompanied with signaling ${ }^{40}$. Considering that changes in glycan content and localization in the mouse cardiac tissue were different in each region, the influence that each cell receives during aging may differ in individual cardiac regions with various cells in vivo.

As the glycan change indicated by GSL-IB4, it is quite possible that molecular changes on the tissue sections shown by glycan profiling may well reflect the specific molecular changes of a particular cell type. However, lectin microarray analysis for tissue would not necessarily agree with the results of lectin staining, since tissue contains multiple glycoconjugate-molecules. To understand the molecular changes in details, it is necessary to collect glycoproteins via lectins and to identify glycans and glycopeptides by LC-MS ${ }^{51}$. On the other hand, in a single-cell and nuclei transcriptomic analysis carried out recently, each cell-specific change was shown to occur in multiple cell 
types and organs along with age-related changes ${ }^{52,53}$. A combination of a single-cell transcriptomic atlas and research on cell-construction or function has been reported. Similar to a single-cell and nuclei transcriptomics research, a glycan atlas can be combined with various cellular analyses. Validating spatiotemporal changes during aging in human cardiac tissues using glycans based on the present study will be helpful for clearing cardiac alterations in age-related disease. In the future, it will be necessary to investigate further structural details of glycan changes and clarify the correlation between molecular changes at each region and functional alterations/disease onset.

The present study reports changes in glycan profiles depending on cardiac localization. We found that the rate of glycan profile changes differs in terms of cardiac localization, and that the amount of sialic acid residues and $\alpha$ galactose residues decrease in the papillary muscle or left ventricular wall. As detection of $\alpha$-galactose residues showed a reduction in microvessels, the structure or the amount of glycans can indicate cardiac tissue conditions. From a clinical perspective, validation of spatiotemporal changes during aging will become more and more important.

\section{Methods}

Tissue section preparation. All animal experiments were approved by the Animal Ethics and Experimentation Committee of Tokyo Metropolitan Institute of Gerontology (TMIG) (No. 17020), and all methods were performed at TMIG in accordance with the relevant guidelines and regulations. C57BL/6N female mice (2-, 6-, 12-, 24-month-old; $\mathrm{n}=4,3,4,4)$ were purchased from Japan SLC, Inc. (Shizuoka, Japan), and reared in the TMIG animal facility for aging. All animals were anesthetized with $50 \mathrm{mg} / \mathrm{kg}$ pentobarbital sodium salt via intraperitoneal injection and sacrificed through ongoing perfusion with saline followed by $4 \%$ paraformaldehyde solution in phosphate-buffered saline (PBS) into the heart. Isolated perfused hearts were re-fixed by $4 \%$ paraformaldehyde immediately. The fixed hearts were embedded in paraffin and cut in a horizontal direction into $3-5 \mu \mathrm{m}$ serial sections for immunohistochemistry and $5 \mu \mathrm{m}$ serial sections for lectin microarray analysis (Fig. 1a).

Lectin microarray analysis. Three generations of female mice (2-, 12-, 24-month-old; $\mathrm{n}=3,3,3)$ were used for lectin microarray analysis (Fig. 1a). The hematoxylin-stained paraffin-embedded thin sections were mounted on a polyethylene naphthalate membrane-coated slide. The two serial tissue sections were cut to $0.5 \mathrm{~mm}^{2}$ in the same region using a LMD7000 (Leica Microsystems, Wetzlar Germany) and collected into equal microtubes. After collection, 
proteins from the dissected tissue fragments were extracted as previously described ${ }^{30}$. Briefly, $200 \mu \mathrm{L}$ of $10 \mathrm{mM}$ citrate buffer ( $\mathrm{pH}$ 6.0) was added to each tissue fragment and incubated at $95^{\circ} \mathrm{C}$ for $1 \mathrm{~h}$. A $50 \%$ slurry of $20-\mu \mathrm{m}$ cellulose (Sigma-Aldrich, Co., Louis, MO) was added to the tube, which was then washed twice with PBS. After centrifuging at $20,000 \times g$ for 1 min at $4^{\circ} \mathrm{C}$, the slurry were solubilized with $20 \mu \mathrm{L}$ of PBS containing $0.5 \%$ Nonidet P-40 and sonicated three times each for $15 \mathrm{~s}$. The sonicated solution was incubated on ice for $1 \mathrm{~h}$ and collected by centrifugation at $20,000 \times g$ for $1 \mathrm{~min}$ at $4^{\circ} \mathrm{C}$. The solution was diluted to $20 \mu \mathrm{L}$ with PBS and labeled with $10 \mu \mathrm{g}$ of Cy3 mono-reactive dye (GE Healthcare, Buckinghamshire, UK) at room temperature for $1 \mathrm{~h}$ in the dark. The reaction solution was adjusted to $100 \mu \mathrm{L}$ with probing buffer (Tris-buffered saline containing $1 \%$ Triton $\mathrm{X}-100,1 \mathrm{mM} \mathrm{CaCl}_{2}$, and $1 \mathrm{mM} \mathrm{MnCl} 2, \mathrm{pH} 7.4)$ and incubated at room temperature for $2 \mathrm{~h}$. The Cy3-labeled solution $(60 \mu \mathrm{L})$ was applied to a LecChip (GlycoTechnica, Yokohama, Japan). After incubating at room temperature for approximately $17 \mathrm{~h}$, the LecChip was washed three times with probing buffer before analysis with the evanescent-field fluorescence scanner GlycoStation $^{\mathrm{TM}}$ Reader 1200 (GlycoTechnica). All data were analyzed using GlycoStation ${ }^{\mathrm{TM}}$ Tools Signal Capture 1.0 and GlycoStation ${ }^{\mathrm{TM}}$ Tools Pro 1.0 (GlycoTechnica). Scanning data were selected under appropriate gain conditions, which satisfied fewer than 63,000 net intensities of all signals, and averaged data were analyzed for glycan characterization. For accurate comparative analysis, the data were used with average-normalization ${ }^{54}$.

Immunohistochemistry, lectin staining, and quantitative analysis. To validate the morphology, all deparaffinized and activated $3 \mu \mathrm{m}$ tissue sections were stained with hematoxylin-eosin (HE). Tissue staining was performed according to the manufactures' instructions. Five micrometer-thick deparaffinized sections were stained with Triticum vulgaris agglutinin (WGA; wheat germ agglutinin) or a combination of Griffonia simplicifolia lectin I (GSL-I) B4 and anti-alpha smooth muscle actin ( $\alpha$-SMA) antibody for phenotype analysis. Briefly, deparaffinized slices were activated using an autoclave with Histofine Deparaffinizing Antigen Activation Solution, pH 6.0, main ingredient is ethylene glycol (\#415281; Nichirei Biosciences Inc., Japan), at $120^{\circ} \mathrm{C}$ for $20 \mathrm{~min}$. Then, tissue sections were washed with PBS, incubated in Biotin Blocking system, setting of $0.1 \%$ avidin solution and $0.01 \%$ biotin solution (\#X059030-2; Agilent Technologies Inc., CA, US) at room temperature each for $10 \mathrm{~min}$, and washed with PBS for $5 \mathrm{~min}$. The sections were incubated with Carbo-Free Blocking Solution (\#SP-5040-125; Vector Laboratories Inc., Burlingame, CA, USA) containing $0.1 \%$ Tween 20 for $1 \mathrm{~h}$. After washing the slides, the tissue slices were allowed to react with the anti- $\alpha$ - 
SMA antibody (\#ab5694; 1:200, Abcam plc, Cambridge, UK) at room temperature for $1 \mathrm{~h}$, or $10 \mu \mathrm{g} / \mathrm{mL}$ of fluorescein isothiocyanate (FITC)-conjugated WGA (\#L4895; SHIGMA Co., Japan) at $4^{\circ} \mathrm{C}$ overnight in PBS. After washing, the sections were incubated with $10 \mu \mathrm{g} / \mathrm{mL}$ of Dylight594-conjugated GSL-IB4 (\#DL-1207; Vector Laboratories) in PBS, in combination with anti- $\alpha$-SMA antibody at $4^{\circ} \mathrm{C}$ overnight. After the tissue sections were washed with PBS, they were incubated in a solution containing anti-Rabbit IgG-AF488 (Thermo Fisher Scientific, 1:1000) in PBS, in combination with a mixed solution containing anti- $\alpha$-SMA antibody at room temperature for $1 \mathrm{~h}$. After washing with PBS, the cells were stained with DAPI for $10 \mathrm{~min}$ and then mounted with the Fluorescent Mounting Medium (Agilent Technologies). The stained sections were observed using a NanoZoomer (Hamamatsu Photonics, Japan) for HE staining, Fluoview (Olympus, Japan) and BZ-X710 (Keyence, Japan) for WGA and a combination of $\alpha$-SMA and GSL-IB4 staining, and then analyzed for the hybrid cell count using the BZ-X710 software (Keyence). Detection of microvessel was performed in the area excluding $\alpha$-SMA staining.

Enzymatic treatment for lectin microarray. Twenty microliters of the reaction solution after Cy3-labeling was digested into sialic acid with sialidase A (\#GK80040; Agilent Technologies) or a2-3sialidase (\#4455; Takara Bio), according to the manufacturer's instructions. The solution was adjusted to $100 \mu \mathrm{L}$ with the probing buffer and incubated at $4{ }^{\circ} \mathrm{C}$ until use. Next, $60 \mu \mathrm{L}$ of the solution was applied to the lectin microarray. The data obtained at 2and 24-month of age were compared with those without enzymatic reactions.

Statistical analysis. Lectin microarray data were analyzed using hierarchical clustering and principal component analysis (PCA) by means of pair-wise comparison, using http://lgsun.grc.nia.nih.gov/ANOVA/ (false discovery rate $<0.05)$. The mean value of the lectin microarray data was used for each PCA. The data are presented as the mean \pm standard error of the mean or median with quartile. Significant differences in GSL-IB4 data were evaluated after Kruskal-Wallis test $(p<0.05)$, using IBM SPSS Statics26 (Stats Guild Inc., Japan).

\section{Availability of data and materials}


The data underlying this article are available in the article and its online supplementary material, and in a database at LM-GlycomeAtlas at https://glycosmos.org/lm_glycomeatlas/index (in preparation).

\section{References}

1 Triposkiadis, F., Xanthopoulos, A. \& Butler, J. Cardiovascular aging and heart failure: JACC Review topic of the week. J Am Coll Cardiol 74, 804-813 (2019).

2 Fish, K. M., Mleczko, J. \& Hajjar, R. J. Of mice and men. Circ Res 123, 1109-1111 (2018).

3 Niccoli, T. \& Partridge, L. Ageing as a risk factor for disease. Curr Biol 22, R741-752 (2012).

4 North, B. J. \& Sinclair, D. A. The intersection between aging and cardiovascular disease. Circ Res 110, 1097-1108 (2012).

5 Thiene, G. \& Valente, M. Degenerative, non-atherosclerotic cardiovascular disease in the elderly: a clinicopathological survey. Aging (Milano) 2, 231-244 (1990).

6 French, J. K. et al. Mechanical complications after percutaneous coronary intervention in ST-elevation myocardial infarction (from APEX-AMI). Am J Cardiol 105, 59-63 (2010).

7 Qian, G., Liu, H. B., Wang, J. W., Wu, C. \& Chen, Y. D. Risk of cardiac rupture after acute myocardial infarction is related to a risk of hemorrhage. J Zhejiang Univ Sci B 14, 736-742 (2013).

8 Walts, P. A. \& Gillinov, A. M. Survival after simultaneous left ventricular free wall, papillary muscle, and ventricular septal rupture. Ann Thorac Surg 78, e77-78 (2004).

9 Bader, F., Manla, Y., Atallah, B. \& Starling, R. C. Heart failure and COVID-19. Heart Fail Rev 26, 1 -10 (2021).

10 Lian, J. et al. Analysis of Epidemiological and Clinical Features in Older Patients With Coronavirus Disease 2019 (COVID-19) Outside Wuhan. Clin Infect Dis 71, 740-747 (2020).

11 Long, B., Brady, W. J., Koyfman, A. \& Gottlieb, M. Cardiovascular complications in COVID-19. Am J Emerg Med 38, 1504-1507 (2020).

12 Lan, J. et al. Structure of the SARS-CoV-2 spike receptor-binding domain bound to the ACE2 receptor. Nature 581, 215-220 (2020).

13 Shang, J. et al. Structural basis of receptor recognition by SARS-CoV-2. Nature 581, 221-224 (2020). 
Wang, Q. et al. Structural and Functional Basis of SARS-CoV-2 Entry by Using Human ACE2. Cell 181, 894-904 e899 (2020).

15 Wrapp, D. et al. Cryo-EM structure of the 2019-nCoV spike in the prefusion conformation. Science 367, 1260-1263 (2020).

Yan, R. et al. Structural basis for the recognition of SARS-CoV-2 by full-length human ACE2. Science 367, 1444-1448 (2020).

Litvinukova, M. et al. Cells of the adult human heart. Nature 588, 466-472 (2020).

Elder, S. S. \& Emmerson, E. Senescent cells and macrophages: key players for regeneration? Open Biol 10, 200309; 10.1098/rsob.200309 (2020).

He, S. \& Sharpless, N. E. Senescence in Health and Disease. Cell 169, 1000-1011 (2017).

Song, P., An, J. \& Zou, M. H. Immune Clearance of Senescent Cells to Combat Ageing and Chronic Diseases. Cells 9, 671; 10.3390/cells9030671 (2020).

Childs, B. G., Li, H. \& van Deursen, J. M. Senescent cells: a therapeutic target for cardiovascular disease. J Clin Invest 128, 1217-1228 (2018). Endo, T. Glycobiology of alpha-dystroglycan and muscular dystrophy. J Biochem 157, 1-12 (2015). Kuno, A. et al. Development of a Data-mining System for Differential Profiling of Cell Glycoproteins Based on Lectin Microarray. Journal of Proteomics \& Bioinformatics 1, 068-072 (2008). Pilobello, K. T., Slawek, D. E. \& Mahal, L. K. A ratiometric lectin microarray approach to analysis of the dynamic mammalian glycome. Proc Natl Acad Sci U S A 104, 11534-11539 (2007).

Itakura, Y., Kuno, A., Toyoda, M., Umezawa, A. \& Hirabayashi, J. Podocalyxin-targeting comparative glycan profiling reveals difference between human embryonic stem cells and embryonal carcinoma cells. Glycomics \& Lipidomics S5, 004; 10.4172/2153-0673.S5-004 (2013). Itakura, Y. et al. $\mathrm{N}$ - and $O$-glycan cell surface protein modifications associated with cellular senescence and human aging. Cell Biosci 6, 14; 10.1186/s13578-016-0079-579 (2016).

27 Miura, Y. et al. Characteristic glycopeptides associated with extreme human longevity identified through plasma glycoproteomics. Biochim Biophys Acta Gen Subj 1862, 1462-1471 (2018). Matsuda, A. et al. Development of an all-in-one technology for glycan profiling targeting formalin- 
embedded tissue sections. Biochem Biophys Res Commun 370, 259-263 (2008).

Matsuda, A. et al. Assessment of tumor characteristics based on glycoform analysis of membrane-tethered MUC1. Lab Invest 97, 1103-1113 (2017).

Zou, X. et al. A standardized method for lectin microarray-based tissue glycome mapping. Sci Rep 7, 43560; $10.1038 /$ srep43560 (2017).

31 Konishi, Y. \& Aoki-Kinoshita, K. F. The GlycomeAtlas tool for visualizing and querying glycome data. Bioinformatics 28, 2849-2850 (2012). Nagai-Okatani, C. et al. LM-GlycomeAtlas Ver. 1.0: A Novel Visualization Tool for Lectin MicroarrayBased Glycomic Profiles of Mouse Tissue Sections. Molecules 24; 10.3390/molecules24162962 (2019). Itakura, Y., Nakamura-Tsuruta, S., Kominami, J., Tateno, H. \& Hirabayashi, J. Sugar-Binding Profiles of Chitin-Binding Lectins from the Hevein Family: A Comprehensive Study. Int J Mol Sci 18; 10.3390/ijms18061160 (2017). Ding, P. et al. Cardiac myosin light chain kinase is necessary for myosin regulatory light chain phosphorylation and cardiac performance in vivo. J Biol Chem 285, 40819-40829 (2010).

Kleipool, E. E. et al. Frailty in older adults with cardiovascular disease: cause, effect or both? Aging Dis 9 , 489-497 (2018).

Wu, J., Xia, S., Kalionis, B., Wan, W. \& Sun, T. The role of oxidative stress and inflammation in cardiovascular aging. Biomed Res Int 2014, 615312; 10.1155/2014/615312 (2014). disease: from mechanisms to therapy. Nat Med 21, 1424-1435 (2015). Sheydina, A., Riordon, D. R. \& Boheler, K. R. Molecular mechanisms of cardiomyocyte aging. Clin Sci (Lond) 121, 315-329 (2011). Rienks, M., Papageorgiou, A. P., Frangogiannis, N. G. \& Heymans, S. Myocardial extracellular matrix: an ever-changing and diverse entity. Circ Res 114, 872-888 (2014).

40 Contessotto, P. et al. Distinct glycosylation in membrane proteins within neonatal versus adult myocardial tissue. Matrix Biol 85-86, 173-188 (2020).

41 Nagai-Okatani, C. \& Minamino, N. Aberrant glycosylation in the left ventricle and plasma of rats with 
cardiac hypertrophy and heart failure. PLoS One 11, e0150210; 10.1371/journal.pone.0150210 (2016). Sato, Y., Akimoto, Y., Kawakami, H., Hirano, H. \& Endo, T. Location of sialoglycoconjugates containing the Sia(alpha)2-3Gal and Sia(alpha)2-6Gal groups in the rat hippocampus and the effect of aging on their expression. J Histochem Cytochem 49, 1311-1319 (2001).

43 Basso, C. et al. Arrhythmic mitral valve prolapse and sudden cardiac death. Circulation 132, 556-566 (2015).

44 Sultan, I., Aranda-Michel, E., Gleason, T. G., Navid, F. \& Kilic, A. Mitral valve surgery for acute papillary muscle rupture. J Card Surg 33, 484-488 (2018).

45 Itakura, Y., Sasaki, N. \& Toyoda, M. Qualitative and quantitative alterations in intracellular and membrane glycoproteins maintain the balance between cellular senescence and human aging. Aging (Albany NY) 10, 2190-2208 (2018).

46 Sasaki, N., Itakura, Y. \& Toyoda, M. Sialylation regulates myofibroblast differentiation of human skin fibroblasts. Stem Cell Res Ther 8, 81; 10.1186/s13287-017-0534-1 (2017).

47 Tateno, H. et al. alpha2-6 sialylation is a marker of the differentiation potential of human mesenchymal stem cells. Glycobiology 26, 1328-1337 (2016).

48 Emde, B., Heinen, A., Godecke, A. \& Bottermann, K. Wheat germ agglutinin staining as a suitable method for detection and quantification of fibrosis in cardiac tissue after myocardial infarction. Eur J Histochem 58, 2448; (2014).

49 Pandya, K., Kim, H. S. \& Smithies, O. Fibrosis, not cell size, delineates beta-myosin heavy chain reexpression during cardiac hypertrophy and normal aging in vivo. Proc Natl Acad Sci U S A 103, 1686416869 (2006).

50 Kovacic, J. C. et al. Endothelial to mesenchymal transition in cardiovascular disease: JACC State-of-theArt Review. J Am Coll Cardiol 73, 190-209 (2019).

51 Narimatsu, H. et al. Current technologies for complex glycoproteomics and their applications to biology/disease-driven glycoproteomics. J Proteome Res 17, 4097-4112 (2018).

52 Tabula Muris, C. A single-cell transcriptomic atlas characterizes ageing tissues in the mouse. Nature 583, $590-595(2020)$. 
53 Vidal, R. et al. Transcriptional heterogeneity of fibroblasts is a hallmark of the aging heart. JCI Insight 4; 10.1172/jci.insight.131092 (2019).

54 Tateno, H., Kuno, A., Itakura, Y. \& Hirabayashi, J. A versatile technology for cellular glycomics using lectin microarray. Methods Enzymol 478, 181-195 (2010).

\section{Acknowledgments}

We would like to thank Atsuko Seki for her critical suggestions, Maki Yoshida for her technical advice, and Editage (www.editage.com) for English language editing. This work was supported by the Ministry of Education, Culture, Sports, Science and Technology of Japan [grant number 16K19098 to Y.I.] and the National Center for Child Health and Development [grant number 29-1 to M.T.].

\section{Author contributions}

YI designed the overall study, performed the experiments, analyzed the data, and prepared the manuscript. YK prepared the mouse cardiac tissue and $\mathrm{YH}$ prepared the thin sections and performed HE staining. YM and TI contributed to data analysis. $\mathrm{CNO}$ and $\mathrm{AK}$ provided the techniques for laser micro dissection and lectin microarray. NS, MU, YT, and TK provided suggestions. MT designed the study and edited the manuscript. All authors have read and approved the final manuscript.

\section{Additional information}

\section{Competing interests}

The authors declare no competing interests

\section{Code availability}

Not applicable.

\section{Ethics approval and consent to participate}


The animal protocol was approval by the Animal Ethics and Experimentation Committee of Tokyo Metropolitan Institute of Gerontology (TMIG) (No. 17020), and all methods were performed at TMIG in accordance with the relevant guidelines and regulations. This study is reported in accordance with ARRIVE guidelines (https://arriveguidelines.org).

\section{Consent for publication}

Not applicable. 


\section{Figure legends}

Fig. 1 Lectin microarray analysis of glycoproteins in mouse cardiac tissue. a) Schematic illustration of lectin microarray analysis of tissue glycoproteins. The hearts of female mice were cut at around the midportion of the cardiac ventricle. Collected sections were heated with $10 \mathrm{mM}$ citrate buffer ( $\mathrm{pH}$ 6.0) and sonicated with PBS, containing $0.5 \%$ Nonidet P-40, followed by the collection of extracted glycoproteins. b) The short-axis of the female mouse cardiac tissue in the three age groups (2-, 12-, 24-month-old; $n=3,3,3)$ and each region of analyzed glycoproteins (No. 1 and 2; forward sides of left ventricular wall, No. 3 and 4; back sides of left ventricular wall, No. 5 and 6; papillary muscle, and No. 7 and 8; ventricular septum).

Fig. 2 Statistical analysis of lectin microarray data. a) Eight regions of three 2-month-old female mice were individually analyzed using principal component analysis. Open and closed circles represent areas No. 1-4 and 5-8, respectively. Left panel in the bi-plot: Cardiac areas, right panel in the bi-plot: Lectin replications. The right panel shows a magnified view of the squared area in the left panel. b) The average of the lateral and inside left ventricles in three mice per group in 2-, 12-, and 24-month-old mice was determined by hierarchical clustering. c) Eight regions of the average of three mice per group in the 2-, 12-, and 24-month-old mice were analyzed. PC3 represents aging. Closed circle (blue or pink) represents No. 1-4 or 5-8. Color gradients (light to dark) of dots reflect progressive senescence (i.e., young to aged). Left panel in the bi-plot: Cardiac areas; right panel in the bi-plot: Lectin replications.

Fig. 3 Cardiac morphology in aging. a) The short-axis of cardiac tissues from 2- and 24-month-old female mice was stained with hematoxylin-eosin (HE) in the papillary muscle regions on the left and right side, respectively $(n=3$ 4/group). Lower panels show the magnified view of the squared area in the upper panels. Scale bars are 50 and $25 \mu \mathrm{m}$ (upper and lower panels, respectively). b) The short-axis of cardiac tissues of 2- and 24-month-old female mice was stained with WGA (60x objective) in the papillary muscle regions on the left and right side, respectively ( $n=3 /$ group). Lower panels represent the magnified view of the squared area in the upper panels. c) Relative intensities relating to

sialic acid- and galactose-binding lectins in the papillary muscle with sialidase A are shown. The average relative 
intensity of three mice in 2- and 24-month-old mice (upper and lower, respectively) was analyzed individually without or with sialidase (open and closed bar, respectively) (mean \pm standard error of the mean (SEM), $n=3 /$ group). Bar graph represents average signal intensity after normalization to without sialidase. d) Relative intensities of sialic acidand galactose-binding lectins in the papillary muscles with $\alpha 2-3$ sialidase. The average relative intensity of three mice in 2- and 24-month-old mice (upper and lower, respectively) was analyzed individually without or with sialidase (open and closed bar, respectively) (mean \pm SEM, $n=3$ /group). Bar graph represents the average signal intensity after normalization to without sialidase.

Fig. 4 Decrease in the number of cardiac microvessel with aging. a) Papillary muscles were stained for GSL-IB4 (red), and merged with anti- $\alpha-S M A$ antibody (green), and DAPI (blue) signals in samples from 2-, 6-, 12-, and 24-monthold mice (upper and lower, respectively) (60x objective). Analysis was performed in the area excluding $\alpha-S M A$ staining. The values of GSL-IB4-positive area of three female mice with seven to ten spots in every age group are shown in box plot. b) The left ventricular walls were stained for GSL-IB4 (red), and merged with anti- $\alpha$-SMA antibody (green) and DAPI (blue) signals in samples from 2-, 6-, 12-, and 24-month-old mice (upper and lower, respectively). The ratios of GSL-IB4-positive area of three female mice with six to nine spots in every age group are shown in the box plot. Analysis was performed in the area excluding $\alpha$-SMA staining. 
Table

Table 1. Relative intensities relating to sialic acid- and galactose-binding lectins in the papillary muscle.

\begin{tabular}{lllllllllllllllllll}
\multicolumn{1}{c}{ Sialidase A $(+)$} & \multicolumn{11}{c}{$\alpha 2-3$ sialidase $(+)$} \\
\hline Lectins & $2(\mathrm{~m} / \mathrm{o})^{*}$ & & Ave. & $24(\mathrm{~m} / \mathrm{o})$ & & Ave. & $2(\mathrm{~m} / \mathrm{o})$ & & Ave. & $24(\mathrm{~m} / \mathrm{o})$ & & Ave. \\
\hline MAL_I & 0.2 & 0.2 & 0.2 & 0.2 & 0.3 & 0.2 & 0.1 & 0.2 & 0.4 & 0.1 & 0.2 & 0.2 & 0.3 & 0.2 & 0.2 & 0.2 \\
SNA & 0.1 & 0.1 & 0.1 & 0.1 & 0.1 & 0.0 & 0.1 & 0.1 & - & - & - & - & - & - & - & - \\
SSA & 0.1 & 0.2 & 0.2 & 0.2 & 0.2 & 0.2 & 0.2 & 0.2 & - & - & - & - & - & - & - & - \\
TJA-I & 0.1 & 0.2 & 0.2 & 0.2 & 0.4 & 0.4 & 0.4 & 0.4 & - & - & - & - & - & - & - & - \\
ACG & 0.4 & 1.2 & 0.4 & 0.7 & 0.9 & 1.1 & 0.8 & 0.9 & 0.5 & 0.5 & 0.4 & 0.5 & 1.5 & 0.9 & 1.1 & 1.1 \\
MAH & 0.3 & 0.3 & 0.4 & 0.3 & 0.3 & 0.3 & 0.3 & 0.3 & 0.5 & 0.3 & 0.5 & 0.4 & 0.5 & 0.6 & 0.4 & 0.5 \\
WGA & 0.8 & 0.5 & 0.6 & 0.6 & 1.0 & 1.0 & 0.9 & 1.0 & 0.8 & 0.6 & 0.7 & 0.7 & 0.7 & 0.6 & 0.5 & 0.6 \\
LTL & - & - & - & - & - & - & - & - & 3.5 & 3.6 & 2.9 & 3.4 & 1.3 & 1.4 & 1.4 & 1.4 \\
ECA & 7.2 & 9.0 & 8.6 & 8.3 & 1.8 & 2.0 & 1.6 & 1.8 & 16.3 & 7.0 & 9.9 & 11.0 & 4.6 & 4.0 & 2.1 & 3.6 \\
RCA120 & 0.6 & 6.8 & 5.5 & 4.3 & 1.0 & 1.3 & 1.0 & 1.1 & 2.3 & 2.7 & 2.9 & 2.7 & 1.2 & 1.5 & 1.1 & 1.2 \\
WFA & 7.2 & 3.9 & 10.2 & 7.1 & 0.7 & 1.3 & 1.1 & 1.0 & 8.5 & 4.1 & 8.1 & 6.9 & 9.4 & 4.5 & 3.2 & 5.7 \\
\hline
\end{tabular}

The data were averaged after a comparison with the no-sialidase sample $(n=3)$.

*The m/o show month old. 


\section{Figures}

a) Preparation of tissue section

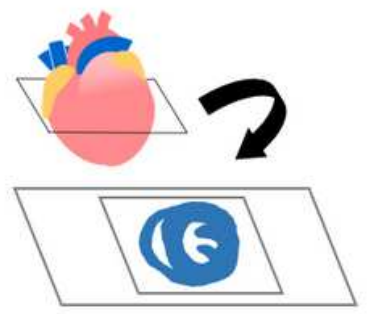

b) 2-month-old

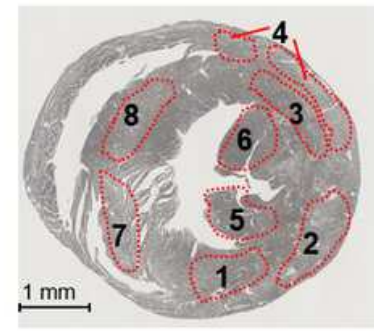

Extraction and labeling of proteins

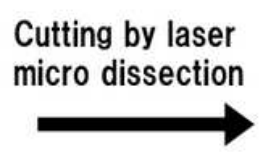

12-month-old

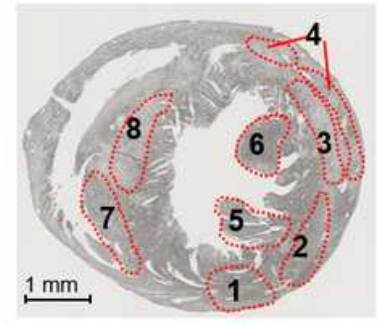

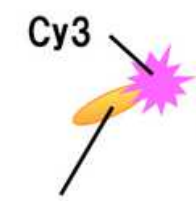

Proteins or glycoproteins

24-month-old

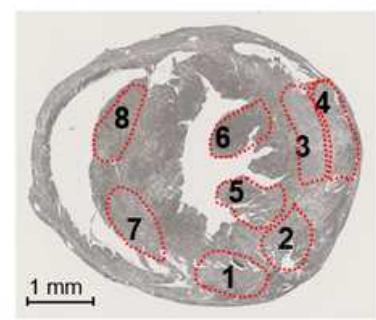

Reaction and detection of glycans

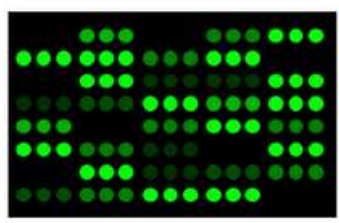

(45 lectins/ 1 well)

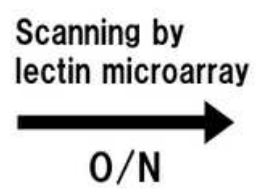

$0 / \mathrm{N}$

Figure 1

Lectin microarray analysis of glycoproteins in mouse cardiac tissue. a) Schematic illustration of lectin microarray analysis of tissue glycoproteins. The hearts of female mice were cut at around the midportion of the cardiac ventricle. Collected sections were heated with $10 \mathrm{mM}$ citrate buffer $(\mathrm{pH} \mathrm{6.0)}$ ) and sonicated with PBS, containing $0.5 \%$ Nonidet P-40, followed by the collection of extracted glycoproteins. b) The short-axis of the female mouse cardiac tissue in the three age groups $(2-, 12-, 24$-month-old; $n=3,3,3)$ and each region of analyzed glycoproteins (No. 1 and 2; forward sides of left ventricular wall, No. 3 and 4; back sides of left ventricular wall, No. 5 and 6; papillary muscle, and No. 7 and 8; ventricular septum). 
a)

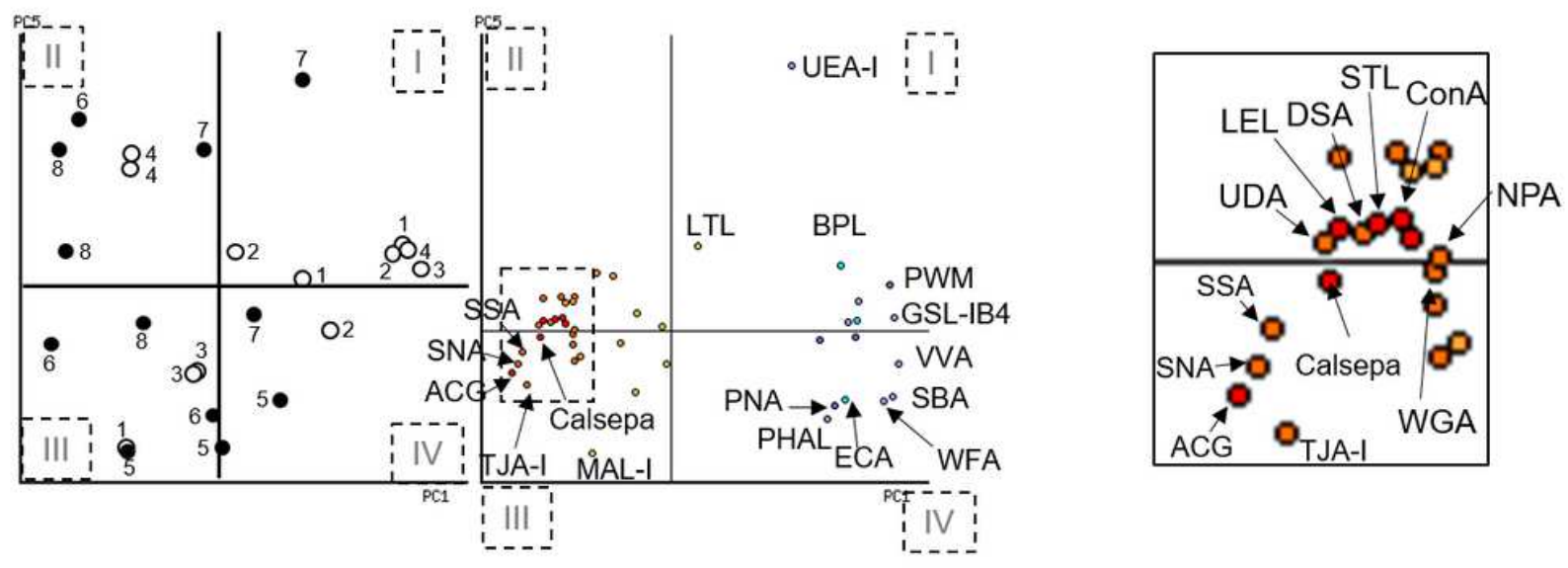

b)

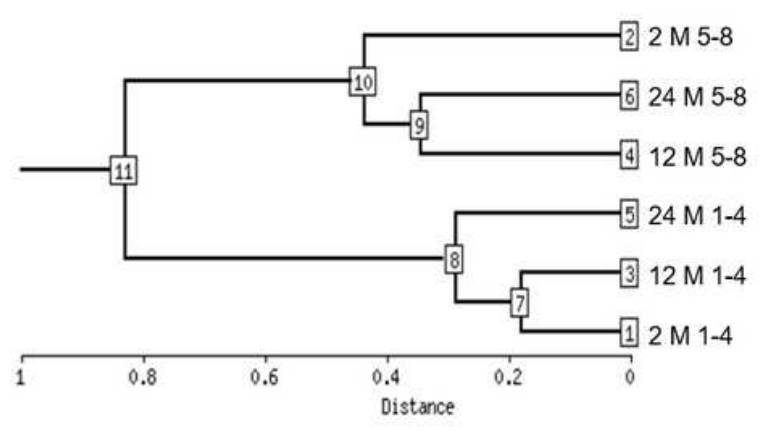

c)

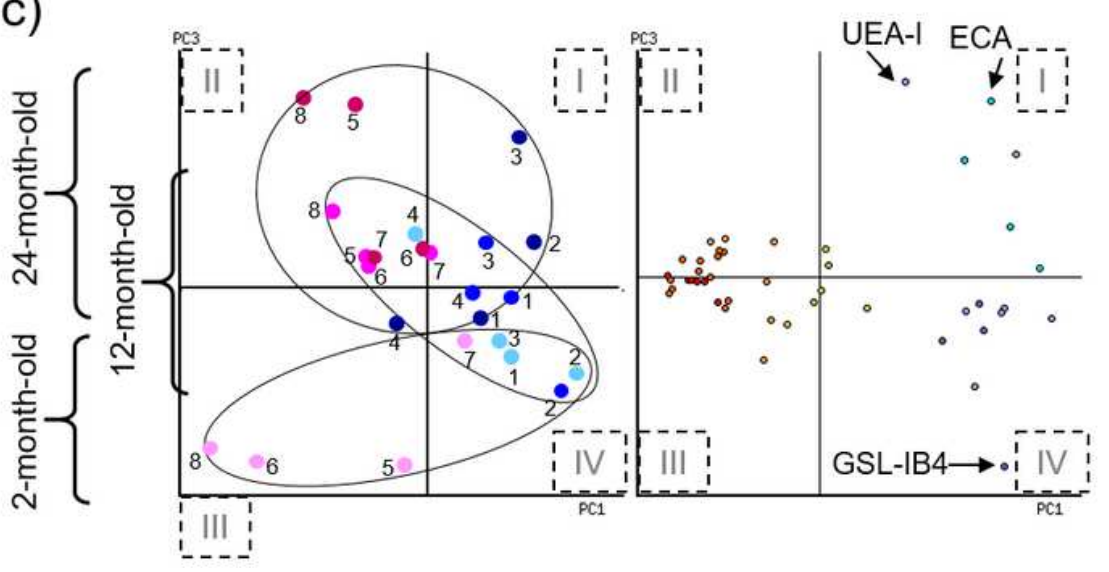

Figure 2

Statistical analysis of lectin microarray data. a) Eight regions of three 2-month-old female mice were individually analyzed using principal component analysis. Open and closed circles represent areas No. 14 and 5-8, respectively. Left panel in the bi-plot: Cardiac areas, right panel in the bi-plot: Lectin replications. The right panel shows a magnified view of the squared area in the left panel. b) The average of the lateral and inside left ventricles in three mice per group in 2-, 12-, and 24-month-old mice was determined by hierarchical clustering. c) Eight regions of the average of three mice per group in the 2-, 12-, and 24-month-old mice were analyzed. PC3 represents aging. Closed circle (blue or pink) represents No. 14 or 5-8. Color gradients (light to dark) of dots reflect progressive senescence (i.e., young to aged). Left panel in the bi-plot: Cardiac areas; right panel in the bi-plot: Lectin replications. 
a)
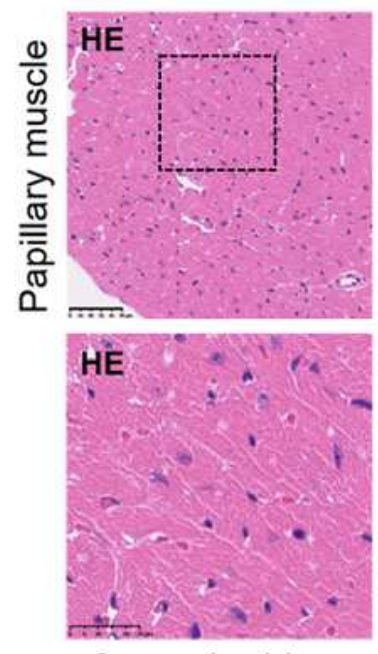

2-month-old
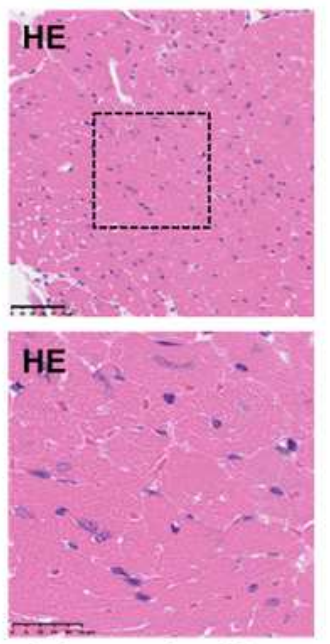

24-month-old b)

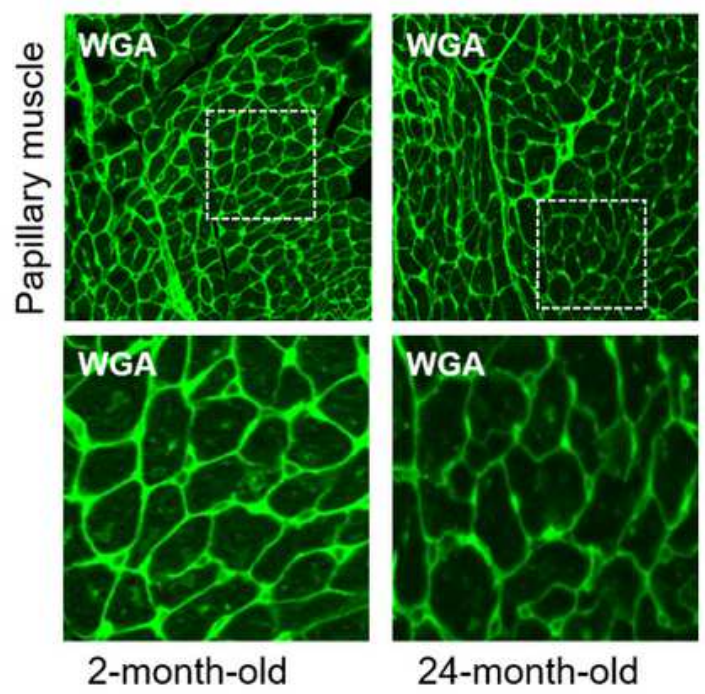

c)
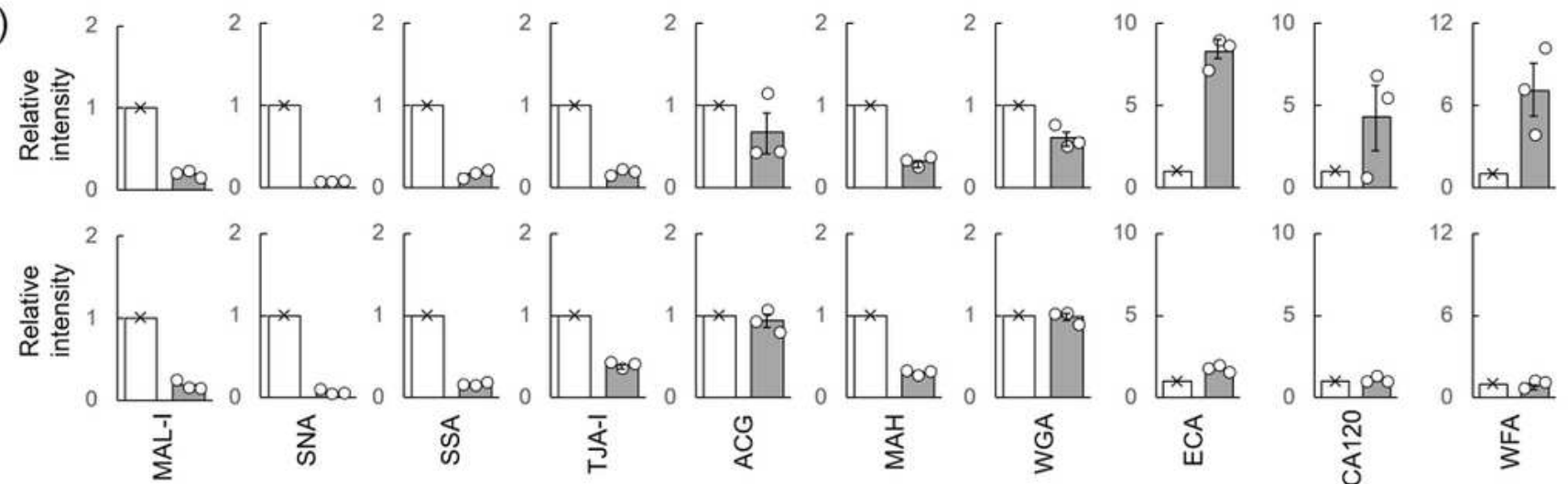

d)

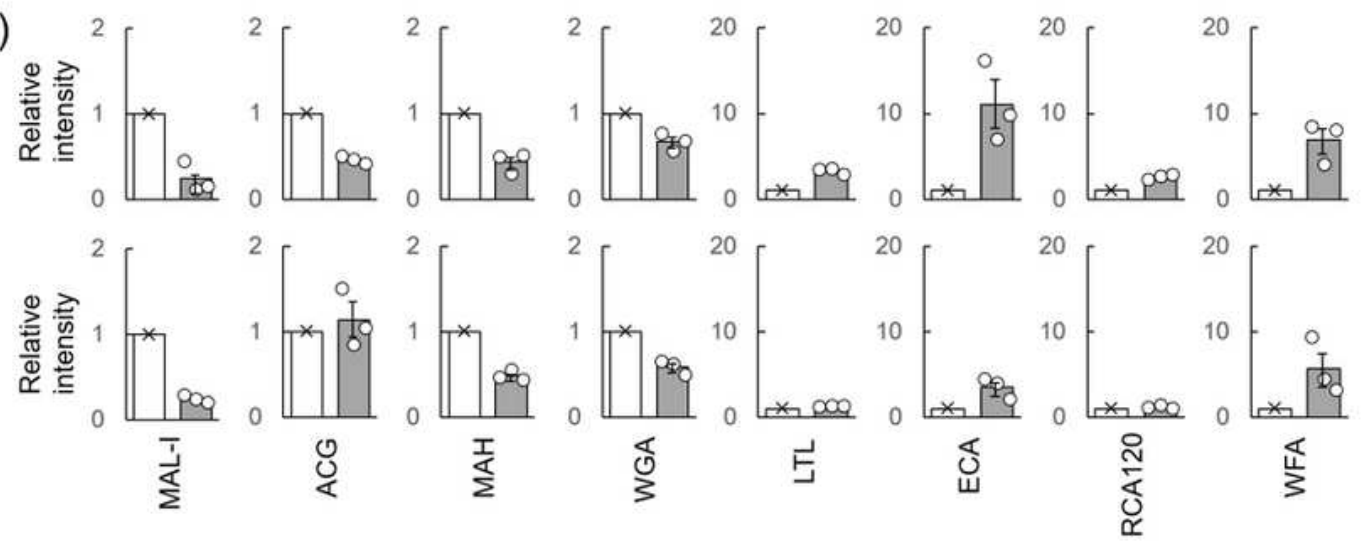

Figure 3

Cardiac morphology in aging. a) The short-axis of cardiac tissues from 2- and 24-month-old female mice was stained with hematoxylin-eosin (HE) in the papillary muscle regions on the left and right side, respectively ( $n=3-4 /$ group). Lower panels show the magnified view of the squared area in the upper panels. Scale bars are 50 and $25 \mu \mathrm{m}$ (upper and lower panels, respectively). b) The short-axis of cardiac tissues of 2- and 24-month-old female mice was stained with WGA (60x objective) in the papillary muscle 
regions on the left and right side, respectively ( $n=3$ /group). Lower panels represent the magnified view of the squared area in the upper panels. c) Relative intensities relating to sialic acid- and galactose-binding lectins in the papillary muscle with sialidase A are shown. The average relative 20 intensity of three mice in 2- and 24-month-old mice (upper and lower, respectively) was analyzed individually without or with sialidase (open and closed bar, respectively) (mean \pm standard error of the mean (SEM), $n=3$ /group). Bar graph represents average signal intensity after normalization to without sialidase. d) Relative intensities of sialic acid and galactose-binding lectins in the papillary muscles with a2-3sialidase. The average relative intensity of three mice in 2- and 24-month-old mice (upper and lower, respectively) was analyzed individually without or with sialidase (open and closed bar, respectively) (mean $\pm S E M, n=3$ /group). Bar graph represents the average signal intensity after normalization to without sialidase.

a)
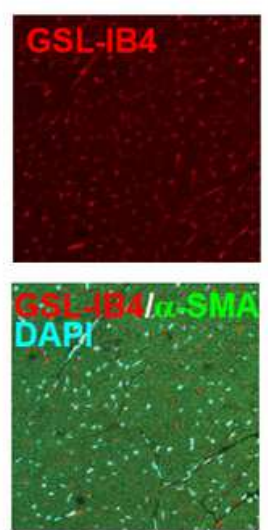

2-month-old

b)
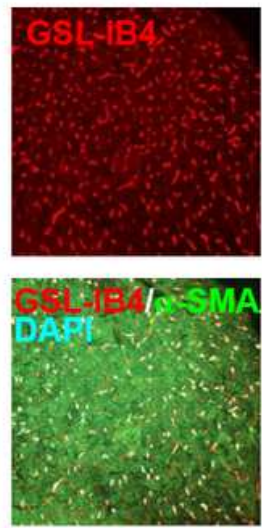

2-month-old
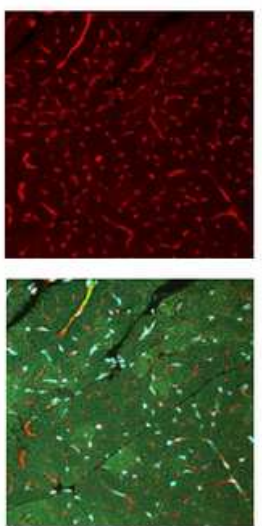

6-month-old
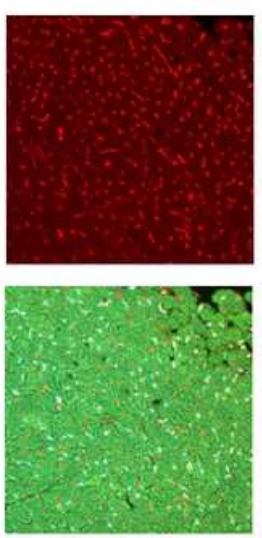

6-month-old
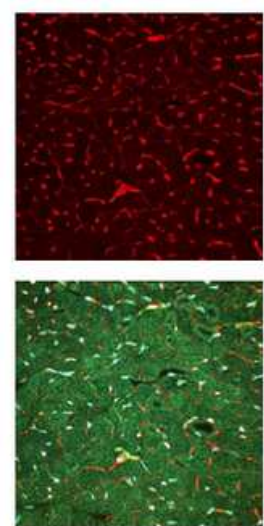

12-month-old
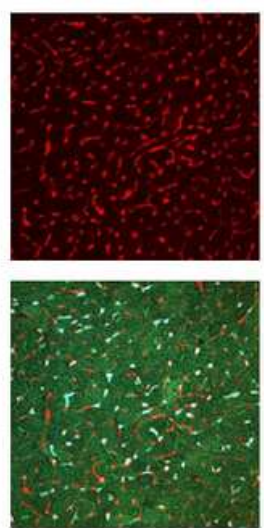

24-month-old
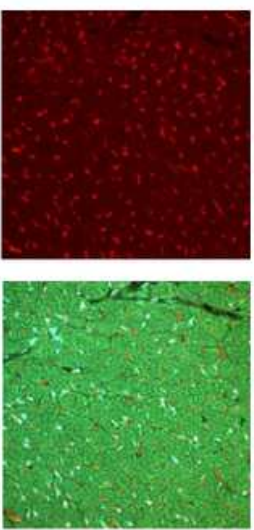

12-month-old
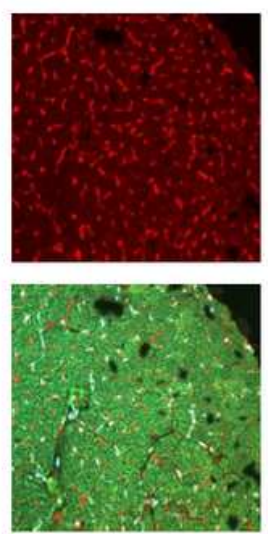

24-month-old

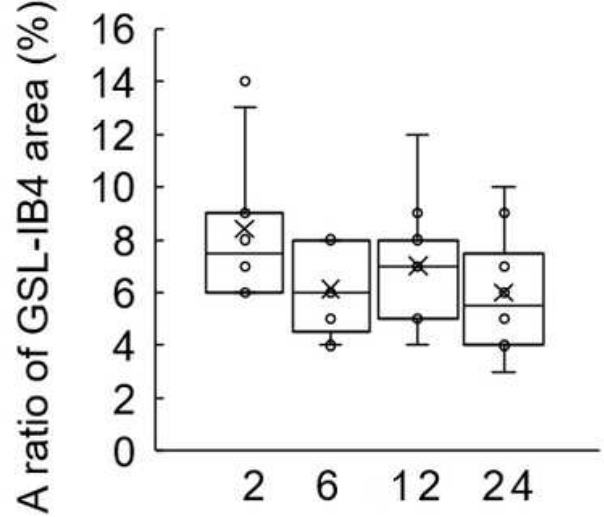

Ages (month-old)

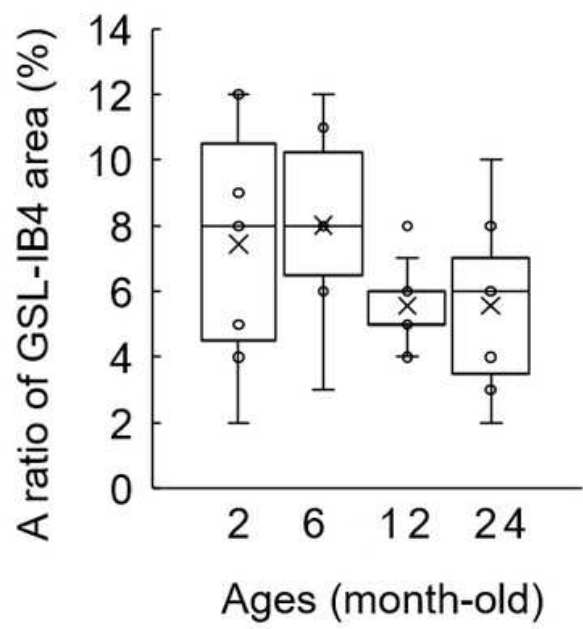

\section{Figure 4}

Decrease in the number of cardiac microvessel with aging. a) Papillary muscles were stained for GSL-IB4 (red), and merged with anti-a-SMA antibody (green), and DAPI (blue) signals in samples from 2-, 6-, 12-, and 24-month old mice (upper and lower, respectively) (60x objective). Analysis was performed in the area excluding a-SMA staining. The values of GSL-IB4-positive area of three female mice with seven to ten spots in every age group are shown in box plot. b) The left ventricular walls were stained for GSL-IB4 
(red), and merged with anti-a-SMA antibody (green) and DAPI (blue) signals in samples from 2-, 6-, 12-, and 24-month-old mice (upper and lower, respectively). The ratios of GSL-IB4-positive area of three female mice with six to nine spots in every age group are shown in the box plot. Analysis was performed in the area excluding a-SMA staining.

\section{Supplementary Files}

This is a list of supplementary files associated with this preprint. Click to download.

- SupplementaryDataScientificReportsItakura.xlsx

- SupplementaryFigureTableScientificReportsItakura.pdf

- SupplementaryFigureLegendsScientificReportsItakura.pdf 